\title{
The use of Dental Operating Microscope for Retrieval of different types of Fractured Implant Abutment Screws: Case Reports
}

\author{
Hend Mahmoud Abou El Nasr* \\ Specialist Senior Registrar, Dubai Health Authority, Endodontics, Dubai, United Arab Emirates
}

\begin{abstract}
The present clinical reports describe the removal of different types of broken implant abutment screws using the endodontic armamentarium with the aid of surgical operating microscope. Endodontic ultrasonic tips and grasping instruments were used under magnification for dislodgment and removal of the broken parts. The internal surfaces of the implants and the screws were visible during manipulation so that no damage affected the internal surfaces of the fixtures. Radiographic examination confirmed complete removal of the fractured parts and magnified examination of their surfaces revealed absence of damage.
\end{abstract}

Keywords: Broken abutment screw; Dental implants; Dental operating microscope

\section{Introduction}

The use of implant-supported prostheses in the replacement of missing natural teeth has become an accepted clinical protocol in dentistry [1]. Implant restorations can fail biologically or mechanically. Biological failures include unsuccessful osseointegration or presence of peri-implantitis; while mechanical failures present as crown fracture, framework fracture, screw loosening, or screw fracture [2]. It was suggested that fractured abutment screws may be replaced by shorter screws without removal of the broken screw remnant as long as three and a half threads are engaged to the internal threads of the dental implant fixture [3]. However, fracture of the implant abutment screw can be a serious problem as the fragment remaining inside the implant may prevent the implant from functioning efficiently. Thus, many methods have been reported for retrieving screw fragments within the implant $[1,4,5]$. Common approaches have been the use of self-made screw- driver, the use of a service set available for the specific implant system, implant repair kits which consist of drills, drill guides, and manual tapping instruments [6-9]. These procedures will not unscrew the fractured fragment. They will cut it into splinters while cutting the threads at the same time, leading to the misfit of the new abutment screw. Moreover, the use of the repair system may not be successful in different situations [5]. Screwing out the fragment is a better method as it will leave the implant threads intact.

The cases described in this article demonstrate the use of the surgical operating microscope and endodontic instruments for the retrieval of 2 different types of broken abutment screws.

\section{Clinical Reports}

\section{Case 1}

A 68 year old male patient was referred to the endodontic department for removal of a broken Ankylos abutment screw from inside a \#42 implant body. The patient originally complained from a loose dental bridge. Upon clinical examination, it was found that the abutment screw in mandibular right lateral incisor was broken; while the implant itself appeared to be well-positioned in all dimensions. The apical part of the screw remained threaded into the implant and was $3 \mathrm{~mm}$ far from the implant neck. A preoperative periapical radiograph (Figure 1A) confirmed that the implant was osseointegrated and showed no signs of peri-implantitis. Two treatment options were explained to the patient: attempting to retrieve the fractured screw, or removing the implant and replacing it with a new one. It was decided that the fractured screw should be removed and the implant restored. An infiltration injection of 2\% lidocaine with 1:80000 adrenaline (Lignospan Special, Septodont, France) was administered to the labial mucosa. Haemostasis was achieved using 25\% aluminium chloride (Alustat gel, Cerkamed, Poland). The implant interior was thoroughly irrigated with saline and dried with paper points. The ultrasonic tip (start X \#3) was used in Satelec handpiece to trough against the screw in a counter-clockwise direction under the surgical operating microscope (SOM) (OPMI pico - Zeiss) to loosen the fractured part. An endodontic explorer was then used in the same direction to dislodge it. The screw came out using this maneuver. A periapical radiograph was taken to confirm the complete retrieval of the broken part (Figure 1B). Another periapical radiograph

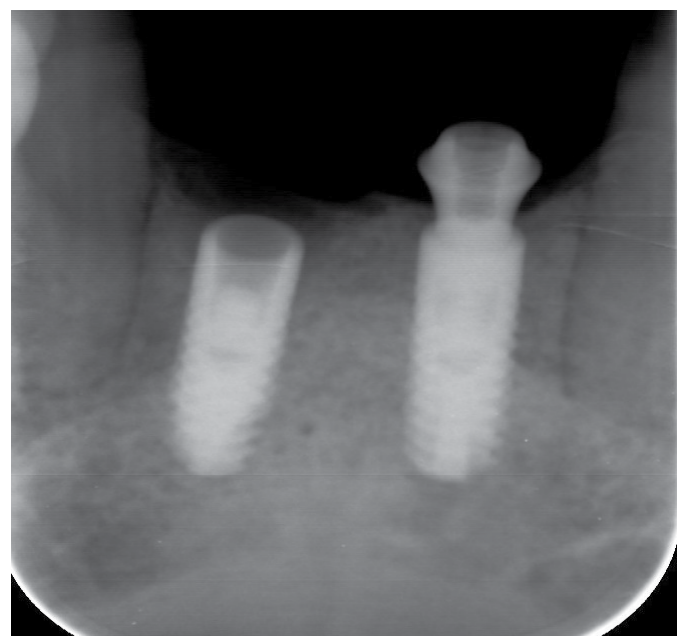

Figure 1A: Periapical radiograph showing the broken screw in implant body in mandibular lateral incisor area.

*Corresponding author: Hend Mahmoud Abou El Nasr, Specialist Senior Registrar, Dubai Health Authority, Endodontics, Dubai, United Arab Emirates, Tel: 00201001539502; E-mail: HMAbouelnasr@dha.gov.ae

Received August 07, 2018; Accepted August 14, 2018; Published August 16, 2018

Citation: Nasr HMAE (2018) The use of Dental Operating Microscope for Retrieval of different types of Fractured Implant Abutment Screws: Case Reports. Dentistry 8: 507. doi:10.4172/2161-1122.1000507

Copyright: (C) 2018 Nasr HMAE. This is an open-access article distributed under the terms of the Creative Commons Attribution License, which permits unrestricted use, distribution, and reproduction in any medium, provided the original author and source are credited. 
Citation: Nasr HMAE (2018) The use of Dental Operating Microscope for Retrieval of different types of Fractured Implant Abutment Screws: Case Reports. Dentistry 8: 507. doi:10.4172/2161-1122.1000507

was taken after securing a gingival former in the implant space (Figure 1C). The broken part was examined under the SOM (Figures 2A-2C) for signs of surface damage. Postoperative and home care instructions were given to the patient, and oral hygiene instructions were reinforced. The fixed bridge was delivered a month later.

\section{Case 2}

A 67 year old male patient was referred to the endodontic clinic for removal of broken abutment screw of size 3.8 integrated Xive implant in mandibular left 2 nd molar area. The patient had a history of fallen porcelain fused to metal crown and he wanted to keep the implant, since it has been in his mouth for 8 years without problems. Clinical examination revealed that the implant site was clean, without signs or symptoms of inflammation or infection. Using the endodontic microscope (OPMI pico, Zeiss, Germany), the implant area was examined, and the screw was viewed after irrigation with sterile saline and dryness with large paper points. Radiographic examination showed 2 broken screws in mandibular left 1st and 2nd molars (Figure 3A). However, the decision by the patient's prosthodontist in agreement with the patient was to retrieve only the screw from within the implant \#37.

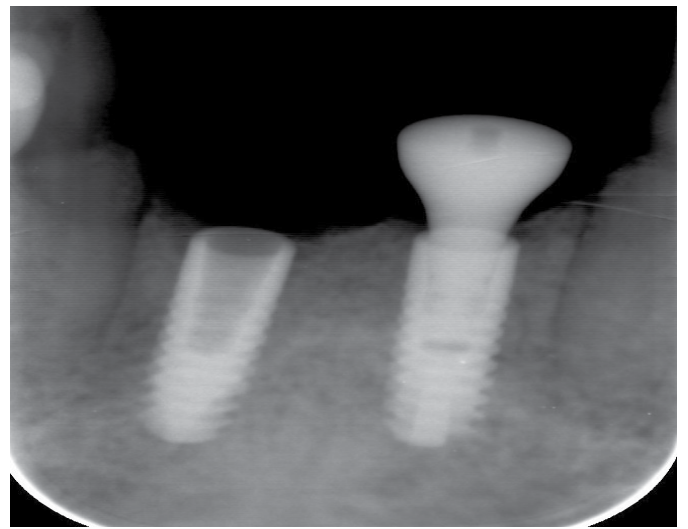

Figure 1B: Periapical radiograph of the implant body after complete removal of the broken fragment.

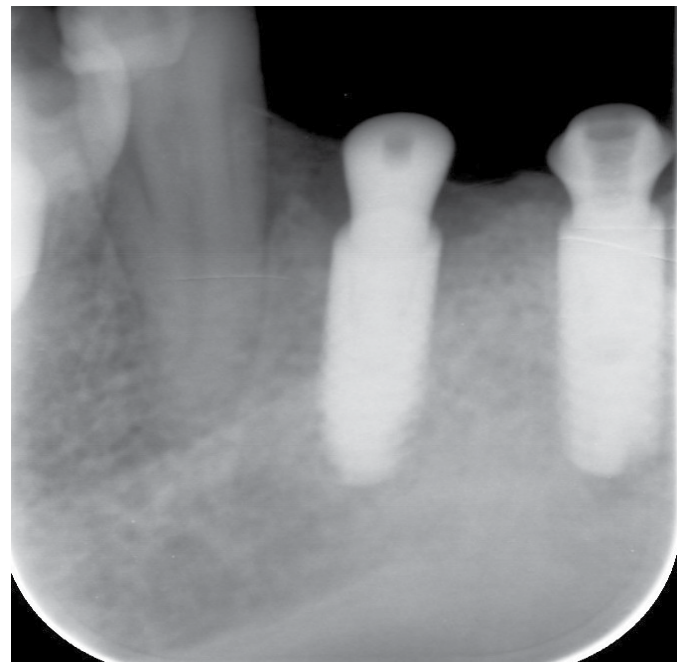

Figure 1C: Periapical radiograph of the implant body with secured gingival former.
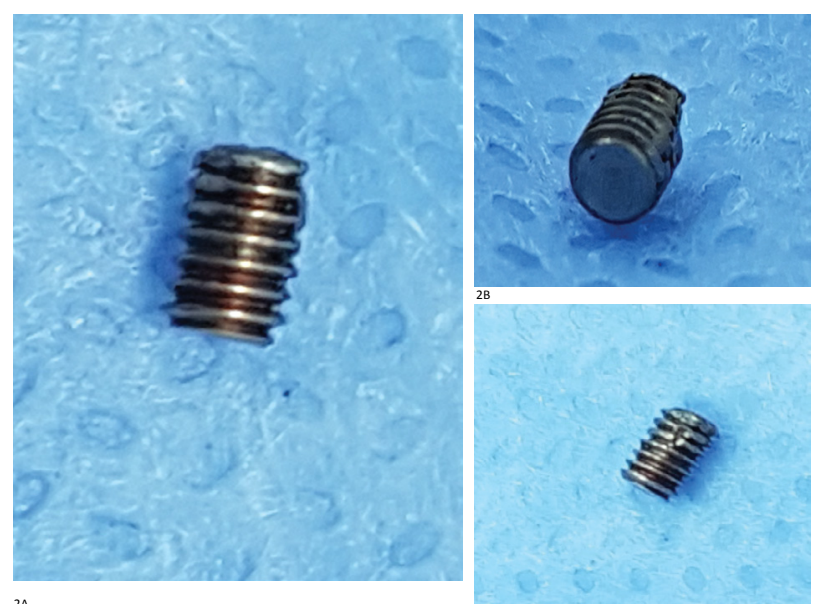

Figure 2A-2C: Photographs for different sides of the retrieved fragment of the broken screws.

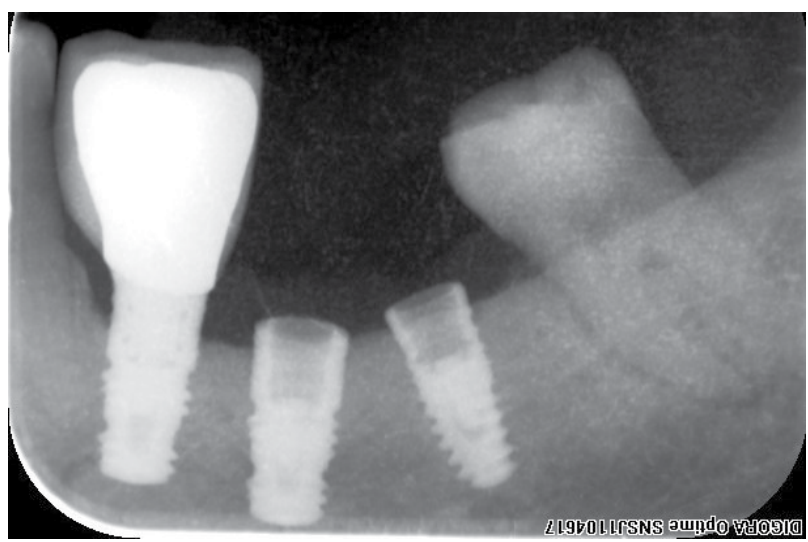

Figure 3A: Periapical radiograph showing the broken screw in implant body in mandibular molar area.

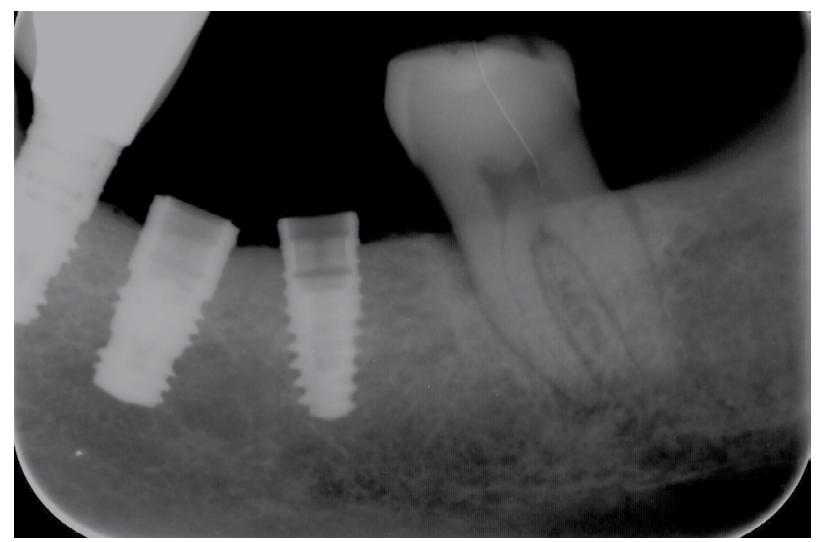

Figure 3B: Periapical radiograph showing the empty space in the body of the implant in the mandibular 2 nd molar site.

An inferior alveolar nerve block of $2 \%$ lidocaine with adrenaline 1:80000 (Lignospan Special, Septodont, France) was administered. 


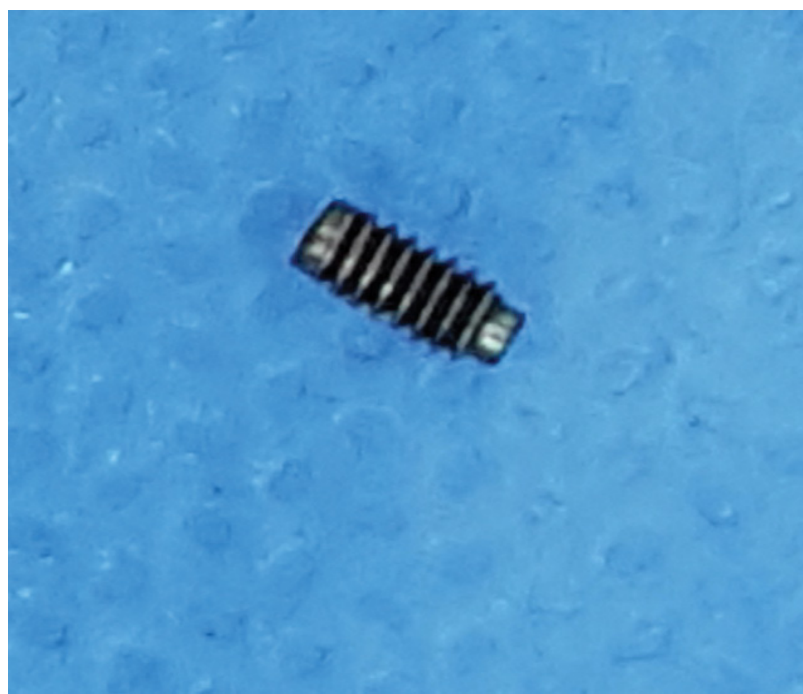

Figure 4: Photograph showing the retrieved Xive abutment screw.

All the following steps were performed under magnification and illumination of the SOM. The Start X ultrasonic tip was used in a counter-clockwise direction in contact with the broken piece of the screw until it felt moving. The screw was displaced in a coronal direction after multiple attempts. The broken piece was then removed using Stieglitz forceps. Postoperative periapical radiograph showed complete removal of the broken part (Figure 3B). Examination of the broken screw revealed its removal without damage to its surface (Figure 4).

Postoperative and home care instructions were given to the patient, and oral hygiene instructions were reinforced. Two weeks later, lower final impression was taken for fixture level for Xive implant \#37. This was followed by delivery of a new implant supported crown for \#37.

\section{Discussion}

The survival rates for implants and implant-supported fixed restorations are reported to be high. Nonetheless, technical complications, namely screw loosening or screw fracture, are not infrequent [10-13]. The primary reason for screw fracture is undetected screw loosening which can be due to bruxism, an illfitting superstructure, or overloading. Other possible reasons for screw loosening include reduced interocclusal distance, a defect in the screw itself, that the screw may have been overtorqued on insertion, or that the abutment may not have been fully seated when the torque was applied to the screw, resulting in increased stress [2,12]. Several techniques for removal of a broken abutment screw have been documented, including screw retrieval systems supplied by various implant manufacturers. However, these methods can involve the risk of damaging the threads inside the implant. Furthermore, the methods employed to grasp the broken fragments or screws are determined according to the location of the fractured abutment -above or below the head of the implant. If an abutment screw fractures above the head of the implant, an explorer, a straight probe or hemostats might be successful [14], but if the screw fracture occurs below the head of the implant, other methods are required [1]. In fact, endodontic ultrasonic tips were first used in both reported cases because the broken fragments remained within the implant body. After witnessing the fragment moving, the endodontic explorer was used in the 1st case, while in the 2nd case, the Stieglitz forceps was used to grasp the fractured part of the screw; however, this was only possible after dislodgment of the broken fragment. The clinical cases reported in the present article were performed by an endodontist (the author), using exclusively endodontic armamentarium under magnification and illumination of the surgical operating microscope which is a tool that is becoming routinely used in endodontics for the management of complicated cases, notably the retrieval of broken instruments inside root canals. The use of SOM allowed precise and accurate removal of the broken pieces of the screws without affecting the internal threads of the implants. The technique was applied to various implant systems and can be effectively incorporated into those clinical situations in which the broken screws are not retrievable by conventional methods.

\section{Conclusion}

The use of SOM is a valuable addition when retrieval of broken screws in dental implants is considered.

\section{Conflict of interests}

The author acknowledges that there is no conflict of interests.

\section{References}

1. Gooty JR, Palakuru SK, Guntakalla VR, Nera M (2014) Noninvasive method for retrieval of broken dental implant abutment screw. Contemp Clin Dent 5: 264-267.

2. Schwarz MS (2000) Mechanical complications of dental implants. Clin Ora Impl Res 11(Suppl.): 156-158.

3. Kim BJ, Yeo IS, Lee JH, Kim SK, Heo SJ, et al. (2012) The effect of screw length on fracture load and abutment strain in dental implants with external abutment connections. Int J Oral Maxillofac Implants 27: 820-823.

4. Yoon JH, Lee H, Kim MY (2015) Safe removal of a broken abutment screw with customized drill guide and rotary instrument: A clinical report. J Prosthodont 25: $170-173$.

5. Canpolat C, Ozkurt-Kayahan Z, Kazazoglu E (2014) Management of a fractured implant abutment screw: A clinical report. J Prosthodont 23: 402-405.

6. Nergiz I, Schmage P, Shahin R (2004) Removal of a fractured implant abutment screw: a clinical report. J Prosthet Dent 91: 513-517.

7. Walia MS, Arora S, Luthra R, Walia PK (2012) Removal of fractured denta implant screw using a new technique: a case report. J Oral Implantol 38: 747-750.

8. Kurt M, Güler AU, Duran I (2013) A technique for removal of a fractured implant abutment screw. J Oral Im-plantol 39: 723-725.

9. Shah K, Lee DJ (2016) An alternative approach for the management of fractured implant abutment screws on a mandibular implant-retained overdenture: A clinical report. J Prosthet Dent 115: 402-405.

10. KreissI ME, Gerds T, Muche R, Heydecke G, Strub JR (2007) Technical complications of implant-supported fixed partial dentures in partially edentulous cases after an average observation period of 5 years. Clin Oral Implants Res 18: $720-726$.

11. Jung RE, Pjetursson BE, Glauser R, Zembic A, Zwahlen M, et al. (2008) A systematic review of the 5-year sur-vival and complication rates of implantsupported single crowns. Clin Oral Implants Res 19: 119-130.

12. Shim HW, Yang BE (2015) Long-term cumulative survival and mechanical complications of single-tooth An-kylos implants: focus on the abutment neck fractures. J Adv Prosthodont 7: 423-430.

13. Tey VHS, Phillips R, Tan K (2016) Five-year retrospective study on success survival and incidence of complications of single crowns supported by dental implants. Clin Oral Implants Res 28: 620-625.

14. Stevens PJ, Frederickson DJ, Gress ML (2000) Implant prosthodontics: Clinica and laboratory procedures. 2nd ed. St Louis, (MO): Mosby, Inc 153-165. 